\title{
A performance evaluation test for laser line scanners on CMMs
}

\author{
Nick Van Gestel*, Steven Cuypers, Philip Bleys, Jean-Pierre Kruth \\ Katholieke Universiteit Leuven, Department of Mechanical Engineering, Celestijnenlaan 300b, 3001 Leuven, Belgium
}

\section{A R T I C L E I N F O}

Available online 10 July 2008

Keywords:

Laser line scanner

CMM

Performance testing

Dimensional measuring

\begin{abstract}
A B S T R A C T
This paper presents a performance evaluation test for laser line scanners on 3D coordinate measuring machines (CMMs). Laser line scanners are becoming more popular in recent years, mainly for free form inspection tasks and reverse engineering. Error specification of these scanners is difficult because of many influencing factors like surface quality, surface orientation and scan depth. Therefore, procedures for evaluation and verification of conventional contact probes (e.g. touch-trigger probes) are not appropriate for non-contact laser line scanners. A straightforward test method that uses a planar test artefact is proposed. It enables to identify the influence of in-plane and out-of-plane angle, as well as scan depth on systematic and random errors of the laser scanner. Experimental results show that the tested commercial laser scanner, after calibration, exhibits systematic errors of about $10 \mu \mathrm{m}$.
\end{abstract}

(c) 2008 Elsevier Ltd. All rights reserved.

\section{Introduction}

Today laser line scanners are frequently used as an alternative for tactile probes on CMMs. Instead of touching the surface to determine coordinates, a plane of laser light is projected onto the surface (Fig. 1a). By capturing the shape of the projected intersection line with a digital camera, the coordinates of the points on the measuring surface are determined by triangulation.

Through calibration of the scanner, camera coordinates are linked with coordinates in the plane of laser light. Since the dimensions of the camera are limited, the scanner will also have a limited field of view (Fig. 1b).

The main advantages of laser line scanning probes, in comparison with conventional touch-trigger probes, are the possibility to measure contactless and to capture many points in a short period of time. This makes them especially useful for digitising free form surfaces. Fig. 2 shows a typical application of a laser line scanner: dimensional quality control of free form surfaces.

The most important disadvantages of laser line scanners, at this moment, are the limited accuracy and the strong influence of surface quality on this accuracy. It is very difficult to measure shiny surfaces, e.g. machined steel or aluminium, because diffuse reflection is needed in order to capture the projected laser line with the camera. The latest laser line scanners are better equipped to deal with this problem but the accuracy for shiny surfaces will always be lower than for diffuse reflecting surfaces. Therefore, metal parts are often sprayed with a diffuse reflecting coating before they are measured. This is of course a serious drawback for

\footnotetext{
* Corresponding author. Tel.: +32 16322537; fax: +32 16322987 .

E-mail address: nick.vangestel@mech.kuleuven.be (N. Van Gestel).
}

many applications. Apart from reflection problems, translucency of the surface also influences the results. Many plastic parts are slightly translucent, and so the laser light also slightly penetrates the surface. This problem can again be solved by applying a diffuse reflecting coating on the object. This of course also affects the measurement results.

Today laser line scanners are at least one order of magnitude less accurate than conventional touch-trigger probes. The accuracy of laser line scanners is difficult to define because standardised procedures to evaluate touch-trigger probes are not appropriate for laser line scanners due to important differences between the two. Firstly, laser line scanners are optical contactless probes while touch-trigger probes are mechanical contact probes. Secondly, a laser line scanner is a 2D measuring device while a touch-trigger probe is a contact sensor. The increasing use of laser line scanners on CMMs, but also on portable measuring arms, implies a growing need for reliable accuracy evaluation tests to analyse and improve the accuracy of the scanners.

The quality of pointclouds, obtained from laser line scanners, has been extensively investigated by Lartigue, Contri and Bourdet [1,2]. They introduced four parameters that describe the quality of a pointcloud: density, completeness, noise and accuracy. In this paper only noise and accuracy are considered, since these two are directly linked to the quality of the scanner. The other two depend strongly on the measurement strategy and object.

\section{Artefacts for performance evaluation tests}

Several authors proposed algorithms and artefacts for the calibration of laser line scanners [3-5]. Today, there are almost no 
a

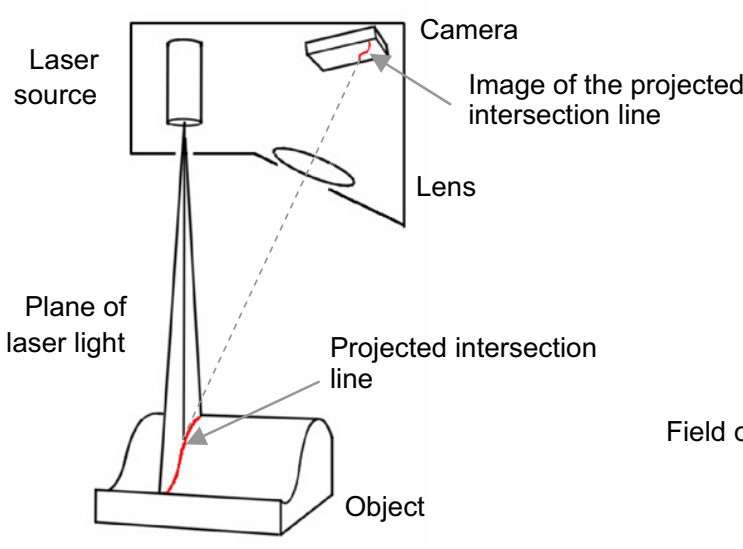

b

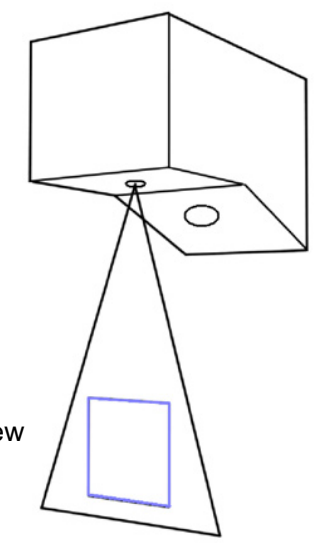

Fig. 1. Principle of laser line scanning (a) and resulting field of view of a laser scanner (b).
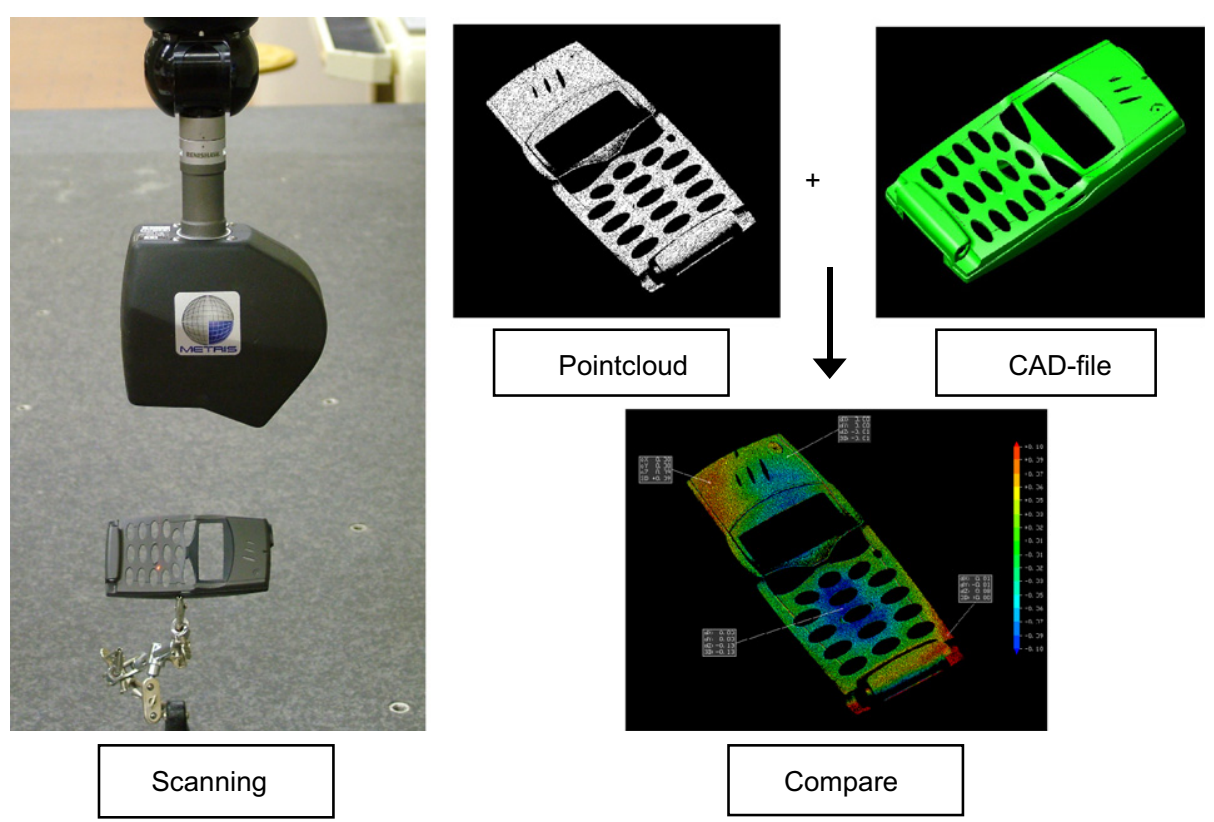

Fig. 2. Typical application of laser line scanners: dimensional quality control of free form surfaces.

publications about performance evaluation tests and artefacts for laser line scanners mounted on CMMs. Possible reference artefacts are illustrated in Fig. 3: single sphere, steps, single or multiple edges, sphere-plane combinations, faceted sphere and doublecurved surfaces.

Often a reference sphere (Fig. 3a) is used as reference artefact. The advantage of using a reference sphere is the accordance to the approach standardised in ISO 10360 for verification of conventional touch-trigger probes and tactile scanning probes, where a sphere is also used. A common reference object is very important for the evaluation of multiple sensor CMMs. However, when the performance of a laser line scanner is evaluated using a reference sphere, results often do not correspond with errors noticed in practice.

Feng proposed a reference sphere mounted on a reference plane (Fig. 3d) to evaluate random and systematic errors of the laser scanner [6]. Others used complex artefacts like double-curved surfaces [7] or a facetted sphere [1]. Several artefacts were tested, but many are too complex and results are difficult to analyse.
When using a sphere, problems occur when only a segment of the sphere is scanned, which is always so when only one scanner orientation is used. Fitting a sphere through a small segment of data leads to unstable results (Fig. 4a). As a result, errors on sphere diameter and position are not an indication of the measurement errors of the scanner; they are rather an indication of an ill-conditioned fitting problem.

When using edge-like artefacts, the variance on tip position can be used as a performance indicator. However, to calculate the tip position, the intersection of 2 fitted lines needs to be calculated and the results are strongly influenced by the data length used to fit the lines. Even more important is the fact that the direction of variance of the intersection point is mainly influenced by the tip angle, independent of the measurement noise direction of the scanner. This is illustrated in Fig. 4b.

A performance evaluation test should be easy, fast and representative for the measurement task. Therefore a simple test method, based on a planar reference, is proposed in this paper. 


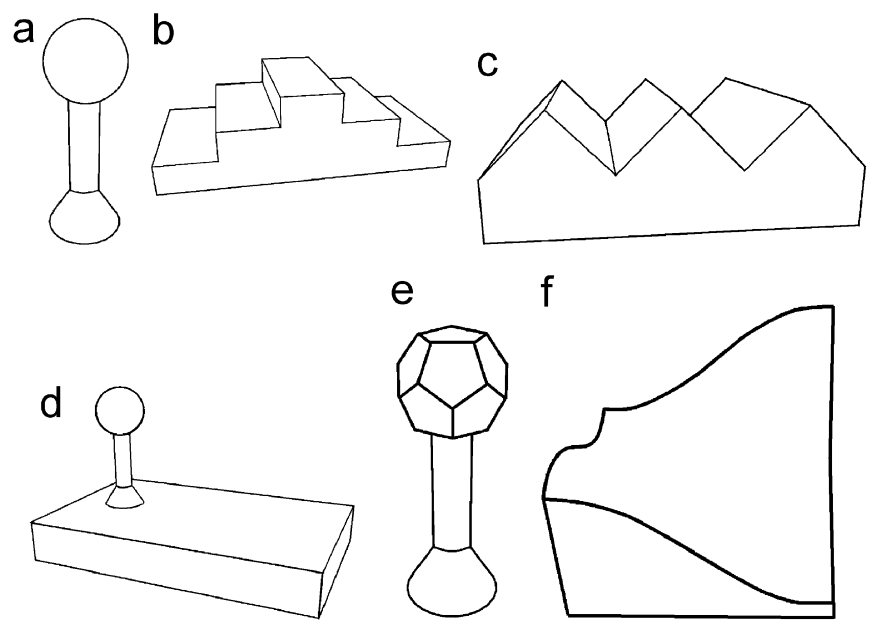

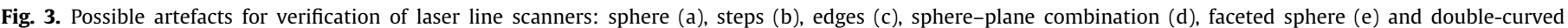
surface (f).
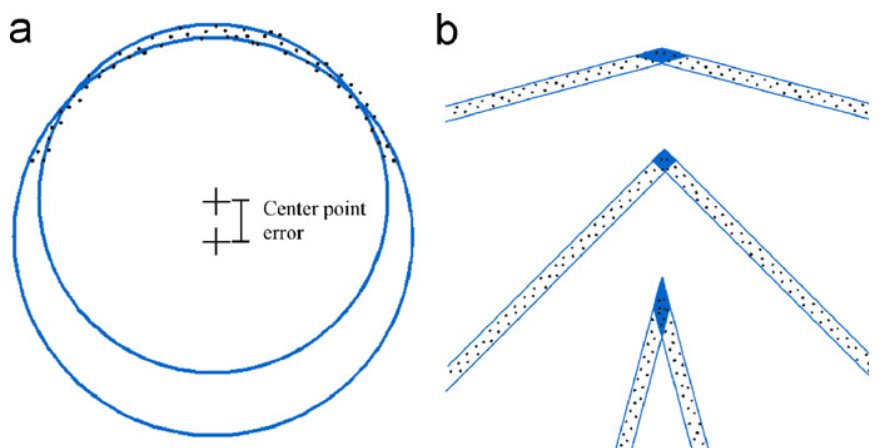

Fig. 4. Fitting a sphere through limited data leads to unstable results (a), and variance on intersection point for edge-like artefacts is determined by tip angle (b).

The next paragraphs will show that this test method enables the user to verify quickly the accuracy of a laser scanner and also to analyse the error sources of the laser line scanner.

\section{Performance evaluation test setup}

The idea of the proposed test setup is straightforward; if points on an object, placed on a CMM, are measured with a scanning probe, the absolute position of these points should be independent of the relative position of the scanning probe with respect to the measured surface. This relative position is determined by the scanning depth (Fig. 5a), the in-plane angle (Fig. 5b) and the outof-plane angle (Fig. 5c) of the scanner, with respect to the surface normal. The results are preferably also independent of surface properties, ambient lighting and time, i.e. the scanner should be stable in time.

The deviation of the absolute location of the measurement points on a reference object, captured with different relative positions of the scanner, can be used as an indication of the measurement error. In order to obtain easily understandable results, the reference object should have a simple form. Therefore, a flat reference plane is used in the test setup. The plane was produced by wire electrical discharge machining (wire EDM) of aluminium, in order to obtain a diffuse reflecting and flat surface (Fig. 6). The flatness of this reference plane is below $5 \mu \mathrm{m}$, measured with 50 points on a Mitotoyo FN905 CMM. The width of the plane is about the same as the scanning width of the scanner $(50 \mathrm{~mm})$, so the plane can be digitised with one linear movement of the scanner over the length of the plane.

The result of such a scan is a planar point cloud through which a least-squares (LSQ) plane can be fitted, using the method described in [8]. The standard deviation of the residual distances $d_{i}$ of the measurement points to the LSQ plane is an indication of the measurement noise of the scanner. When more scans are taken from different scanning positions and orientations, the difference in position of the fitted LSQ planes can be seen as an indication of the systematic error. To calculate the distance, one of the scanned planes is taken as a reference; this can be chosen arbitrarily but is often the first or last scanned plane. Since the centroid of each pointcloud must lie on the fitted LSQ plane [8], the distance $\boldsymbol{D}_{j}$, between the reference and $j$ th planar pointcloud can be calculated as follows:

$D_{j}=\boldsymbol{a} \cdot\left(\overline{\boldsymbol{x}}_{j}-\overline{\boldsymbol{x}}_{r}\right)$

where $\boldsymbol{a}$ is the vector containing the direction cosines of the reference LSQ plane $(|\boldsymbol{a}|=1)$ and $\overline{\boldsymbol{x}}_{r}$ the centroid of the reference pointcloud. $\overline{\boldsymbol{x}}_{j}$ is the centroid of the $j$ th pointcloud.

Fig. 7 illustrates the calculation of the random and systematic error, although Fig. 8a indicates that this reasoning is not always correct. If the reference plane is not flat, or if the scanned pointcloud has a large systematic error component, the standard deviation will not represent the $(1 \sigma)$ measurement noise. Fig. $8 \mathrm{~b}$ shows one of the actually obtained scan results. It is clear that, for this scan result, the measurement noise is the most contributing factor for the standard deviation. This means that the standard 
a

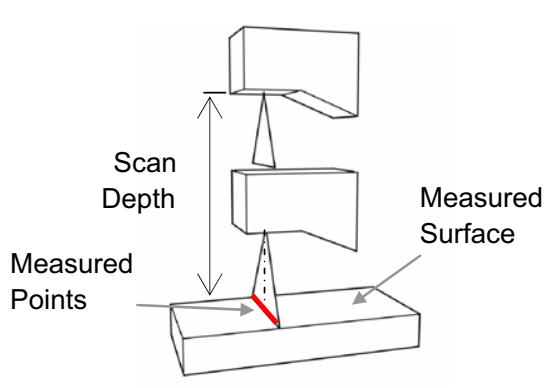

b

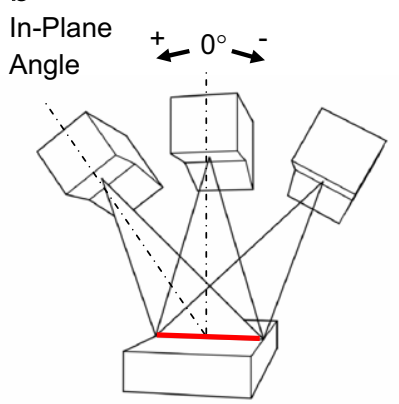

C

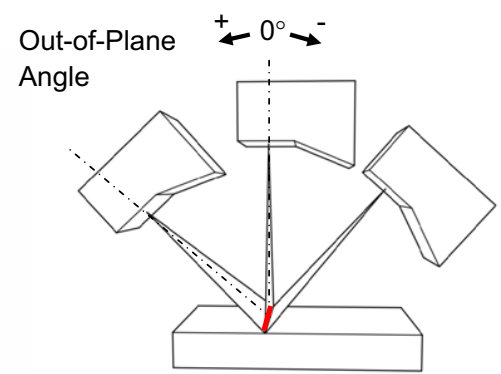

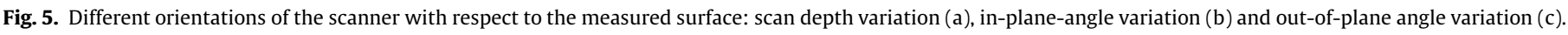

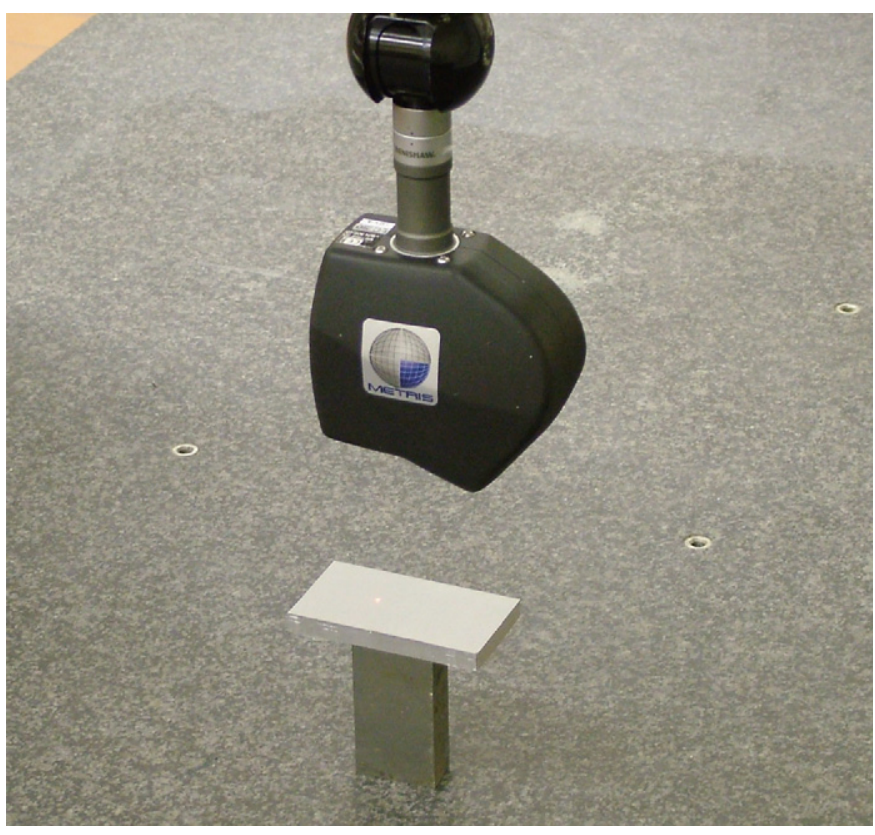

Fig. 6. Picture of the test setup.

deviation is a good indication for the random error or measurement noise.

The tests that are described hereafter are all based on the reasoning described above. First a quick verification test is presented. This test enables to determine, within a few minutes, the accuracy of the scanning process. It also verifies the validity of the scanner calibration. The results quickly give the user an idea of the accuracy of the digitising process. Afterwards a depth test is presented to analyse the influence of the scan depth on random and systematic errors. The influence of the in-plane and out-ofplane angle on the random errors is investigated thereafter. Finally, the evolution of errors in time is investigated through a stability test.

\section{Quick verification test}

This test can be used by the operator to quickly get an idea of the accuracy of the scanner or the validity of the last calibration. The reference plane is scanned under different orientations and in different scanner positions, in fact all orientations and positions with respect to the normal surface that are used for the scanning task. Instead of fitting a LSQ plane through each scan, all scans are put together and one plane is calculated through these points. The size of the residual distances is an indication of the accuracy of the scanner and represents the total error; this includes random and systematic scanner error but also possible errors of the rotation head and positioning errors of the CMM (random and systematic). The result of such a quick verification test is given in Fig. 9.

When it is necessary to include the influence of the surface properties on the scanning accuracy, one can take a reference plane of the same material as the object that has to be scanned. It is not even necessary to have a real flat reference plane. It is also possible to take one of the scans as a reference scan. A surface is generated from this scan by meshing the pointcloud. This surface is then used as a reference surface, with which all the other scans can be compared. The calculated deviations determine the measurement errors of the scanner.

This 'quick verification test' gives an idea of the maximum expected measurement error. This is a very valuable test to evaluate the possible errors of a scanning task. When the reason why the accuracy is out of specification needs to be analysed, or when a qualification of the measurement error is necessary, this test does not give enough information. However, the next sections will demonstrate that, when the scans under different positions are kept separately, this simple test setup is also useful for further analysing the errors of the scanner.

\section{Scan depth test}

The scanning depth test evaluates the influence of the scan depth on the systematic and random error. In order to evaluate this error, the reference plane is scanned at 10 equally spaced scan depth levels; this was done 10 times to get an idea of the repeatability of the results. Level 1 is the position where the scanner is closest to the reference plane, level 10 is the level when the scanner is furthest away from the reference plane, as illustrated in Fig. 10. The distance between level 1 and 10 almost equals the depth of the field of view of the scanner (50 mm).

The standard deviation for each scanned plane and the distances from each plane to the lowest of the 100 measured planes are visualised in Fig. 11. The standard deviation is, as explained in Section 3, an indication of the digitising noise. The distances between the planes are an indication of the systematic errors in function of scanning depth. Since the triangulation angle is becoming smaller with increasing scan depth, the measurement noise should rise with enlarging scan depth. 


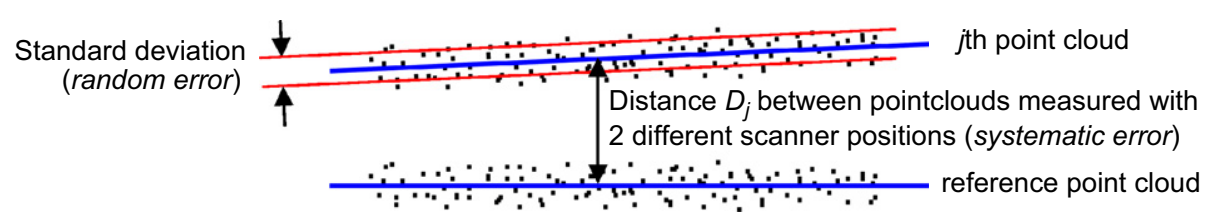

Fig. 7. Determination of random and systematic errors.

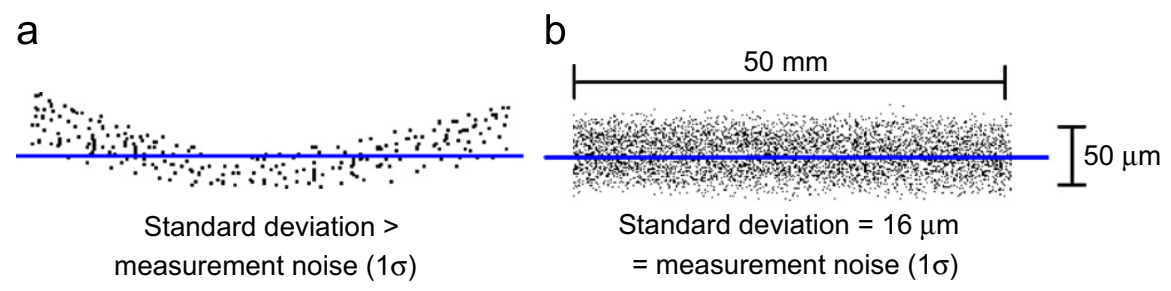

Fig. 8. Shape deviations influencing the calculated standard deviation.

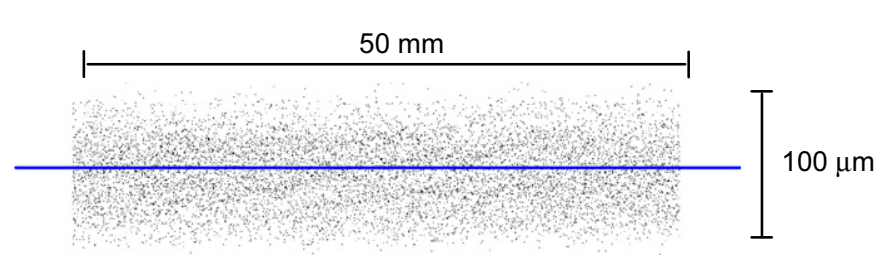

Standard deviation: $23 \mu \mathrm{m}$ Accuracy $(2 \sigma): 46 \mu \mathrm{m}$

Fig. 9. Result of a quick verification test with a plane.

As expected, the digitising noise rises with increasing scan depth. When the scanner is scanning as close to the plane as possible, the standard deviation of the measured plane is about $15 \mu \mathrm{m}$. Since the points have a normal distribution, this means that the distance of $95 \%$ of the measured points to the LSQ plane is within $\pm 30 \mu \mathrm{m}(= \pm 2 \sigma)$. For points measured when the scanner is furthest away from the plane, this is, on average, $\pm 43 \mu \mathrm{m}$. It can be seen that these results are repeatable.

When looking at the distances between the planes it can be seen that, for this test, there was for each curve a systematic difference of about $15 \mu \mathrm{m}$ in distance between planes scanned at level 1 and planes at level 10 . Contrary to the standard deviation, the measured distances are less repeatable. Although the shape of the curves is the same, they are slowly shifting upwards as scanning through the 10 levels is repeated. For level 10 , there is a difference of about $15 \mu \mathrm{m}$ between the first and the last scan. The time difference between the first and the last scan was $30 \mathrm{~min}$. It is also clear that when a scan at level 1 is combined with a scan at level 10 , which is taken 30 min later, there is a systematic difference of $30 \mu \mathrm{m}$ between both pointclouds.

The reason why the results are drifting in time is not totally clear. Although the scanner was already warmed up for some hours when starting the test, the most plausible explanation for the drift is the thermal influence of the heat production of the scanner itself. The stability and warming up effect of the scanner are investigated further in Section 7.

\section{Angle test}

Scan depth determines the location of the surface with respect to the scanner. The in-plane and out-of-plane angle are two

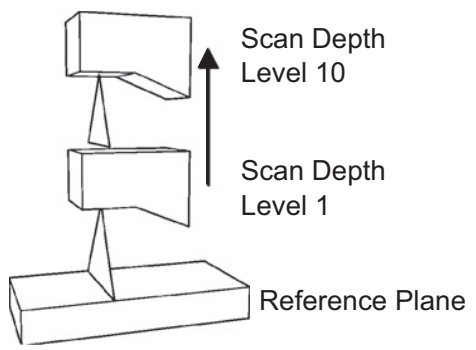

Fig. 10. Reference plane scanned at several scan depths.

parameters determining the orientation of the surface normal with respect to the scanner. These angles can also have an influence on the errors of the scanner. To investigate this influence, the reference plane was scanned while varying the inplane and out-of-plane angles.

Fig. 12 shows the influence of in-plane and out-of-plane angle on the standard deviation of the scanned planes. For the in-plane angle, only positive angles are displayed, due to symmetry reasons, from $0^{\circ}$ to $+60^{\circ}$. The out-of-plane angle is varied from $-45^{\circ}$ to $+60^{\circ}$ (convention displayed in Fig. 5c). While evaluating the influence of one scanning angle, the other angle is kept $0^{\circ}$.

It can be seen that the standard deviation of the scanned plane lowers with increasing angle. For $60^{\circ}$ in-plane and out-of-plane angle, the standard deviation is about half the standard deviation at $0^{\circ}$. This does not mean that the measurement noise lowers with increasing scanning angles, as illustrated in Fig. 13. The measurement noise occurs mainly in the depth direction of the scanner. When the measurement noise is unidirectional, the inclination of the scanned plane is decisive for the resulting standard deviation. It is assumed that this is the main reason why the standard deviation is decreasing with increasing angles.

\section{Thermal stability test-warming-up test}

The results, obtained in Section 5, showed that there is a need to test the stability of the scanner. In order to evaluate this stability, the scanner was calibrated after warming up for several hours. After switching off the scanner and letting it cool down, the scanner was turned on again. Starting from this moment the reference plane was scanned every $3 \mathrm{~min}$ at level 1, 5 and 10 . The 
a

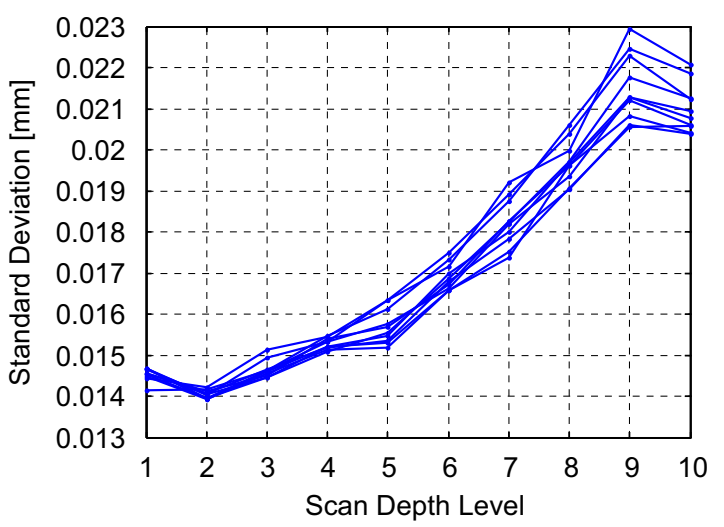

b

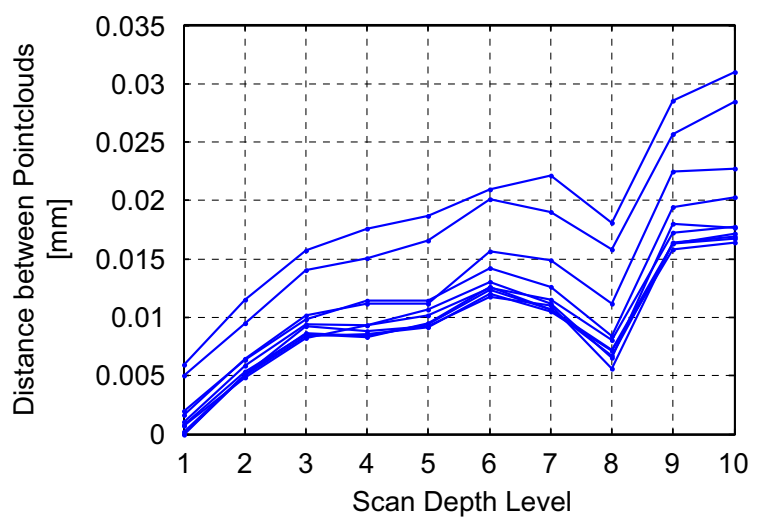

Fig. 11. Influence of scan depth level on random errors (a) and systematic errors (b).

a

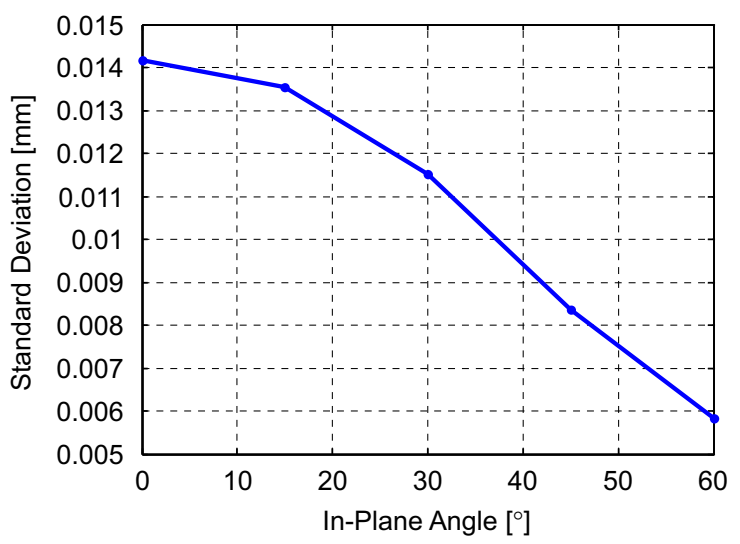

b

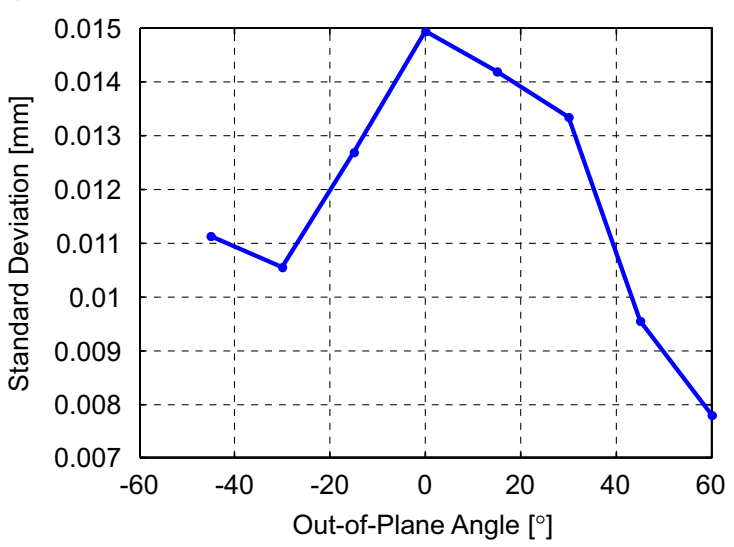

Fig. 12. Influence of in-plane angle (a) and out-of-plane angle (b) on the standard deviation.

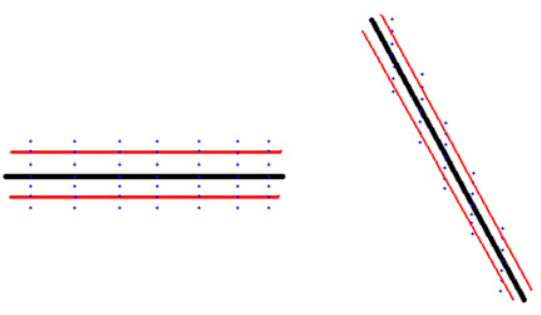

Fig. 13. Scanning angle determining the standard deviation in case of unidirectional noise.

positions of the scanned planes, taken during the tests, are presented in Fig. 14.

This test illustrates that, in the beginning of the test, the difference between the planes, scanned at the lowest and highest position in the range, is about $0.1 \mathrm{~mm}$. This is normal, since the scanner is not warmed up yet. According to the manufacturer's recommendations the scanner should be warmed up after about $30 \mathrm{~min}$. However, from the results it can be seen that it lasts more than $1 \mathrm{~h}$ before level 1, 5 and 10 coincide. Fig. 14 also shows that the results stay drifting several micrometres when the scanner is already warmed up. This drift is also the reason why the curves in Fig. 11 are shifting. This means that the systematic error is only systematic, i.e. stable, during a limited time period. The random error is not illustrated in Fig. 14, but was quite stable in time as could already be seen in Fig. 11.

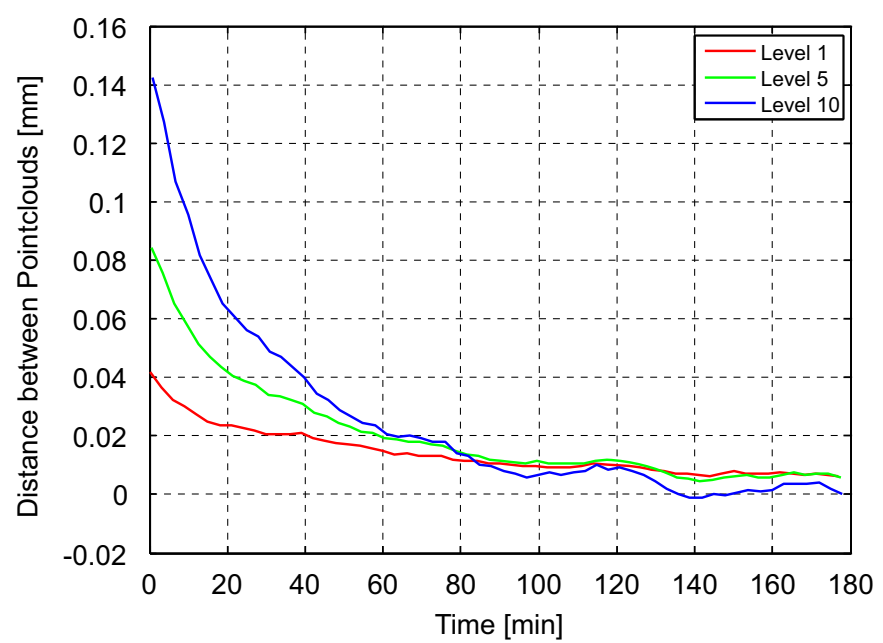

Fig. 14. Stability test results 1 day after scanner calibration.

When the scanner is not recently calibrated, the 'quick verification test' sometimes indicates large errors. Fig. 15a shows a stability test that was done after a large error (due to expired scanner calibration) was noticed in such a quick verification test. This resulted in a systematic error of $120 \mu \mathrm{m}$ after warming up (compared to the normal systematic error of $10-15 \mu \mathrm{m}$ ). This shows that a regular calibration of the scanner is necessary. Errors like these can easily be detected with the quick verification test 
a

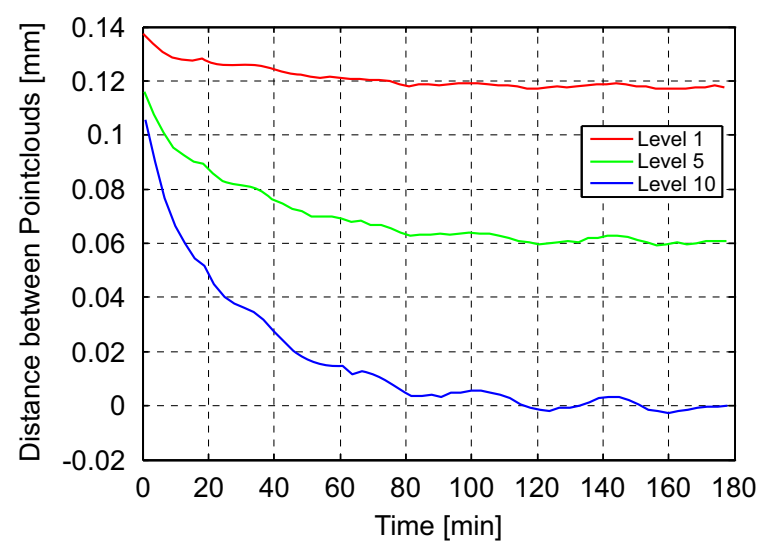

b

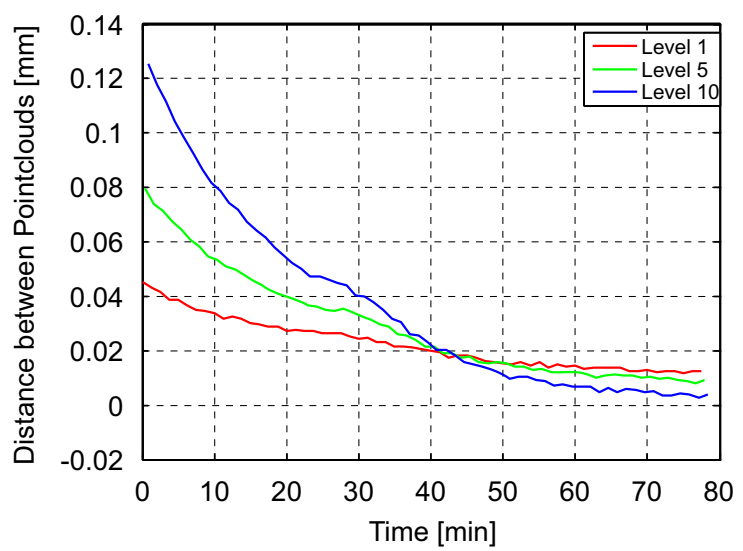

Fig. 15. Stability test results with expired scanner calibration (a) and when calibration was done while scanner was not completely warmed up yet (b).

proposed in Section 4. When calibration is needed, this can be done in situ by the user for most laser line scanners.

It is also important that the scanner is completely warmed up before starting the calibration procedure. Fig. 15b shows the results of a stability test shortly after calibration of the scanner: the scanner was calibrated only after $40 \mathrm{~min}$ warm-up, then switched off and cooled down, and finally switched on again to perform the stability test. The results indicate that after about 40 min there is almost no systematic error (which is normal since the scanner was previously calibrated after $40 \mathrm{~min}$ warm-up). However, after intersecting, the curves separate again, showing that the scanner continues to drift thermally even after $40 \mathrm{~min}$. This means that the scanner was not warmed up completely when doing the calibration. Since those tests, the design of the scanner has been changed to ensure faster warm-up and improve thermal stability.

\section{Conclusion}

In this paper, a straightforward performance evaluation test for laser line scanners is presented. A flat reference plane is scanned under different probe positions and plane orientations. Even though the test setup is very simple, it is possible to obtain a lot of useful information from it, even more than with other complex test methods.

A quick verification test allows to get an idea of possible measurement errors of the scanner (and CMM), just within a few minutes. The proposed test setup also allows to investigate the influence of scan depth, in-plane and out-of-plane angle on random and systematic errors of the scanner. The standard deviation of the scanned reference plane is an indication of the random error (measurement noise) of the scanner. The distance between planes, scanned in different scanner probe positions, is an indication of the systematic error.

The scan depth has an important influence on systematic and random errors of the tested scanner. In-plane and out-of-plane angle have an important effect on the measured standard deviations, because the measurement noise is mainly concentrated in the depth direction of the scanner. A stability test shows that warming-up effects have a major influence on the accuracy of the laser line scanner.

\section{References}

[1] Lartigue C, Contri A, Bourdet P. Digitised point quality in relation with point exploitation. Measurement 2002;32:193-203.

[2] Bourdet P, Contri A, Lartigue C. Quality of 3D digitised points with non-contact optical sensors. Ann CIRP 1999;51(1):443-6.

[3] Xi F, Liu Y, Feng H-Y. Error compensation for three-dimensional line laser scanning data. Int J Adv Manuf Technol 2001;18:211-6.

[4] Che C, Ni J. A ball-target-based extrinsic calibration technique for highaccuracy 3-D metrology using off-the-shelf laser-stripe sensors. Precis Eng 2000;24:210-9.

[5] Wang G, Zheng B, Li X, Houkes Z, Regtien PPL. Modelling and calibration of the laser beam-scanning triangulation measurement system. Robot Autonomous Syst 2002;40:267-77.

[6] Feng H, Liu Y, Xi F. Analysis of digitizing errors of a laser scanning system. Precis Eng 2001;25:185-91.

[7] Savio E, De Chiffre L, Schmitt R. Metrology of freeform shaped parts. Ann CIRP 2007;56(2):810-35.

[8] Shakarji CM. Least-squares fitting algorithms of the NIST algorithm testing system. J Res Natl Inst Stand Technol 1998;103:633-41. 